\title{
Incidental retroperitoneal paraganglioma, imaging workup and CT-guided biopsy: A case report
}

\author{
Michael Hofko ${ }^{1 *}$, Benedikt Hergan ${ }^{2}$, Franz A Fellner ${ }^{1,2}$ \\ ${ }^{1}$ Central Radiology Institute, Kepler University Hospital, Medical Faculty of the Johannes Kepler University, Linz, Austria \\ ${ }^{2}$ Medical Faculty of the Friedrich-Alexander-University of Erlangen-Nürnberg, Erlangen, Germany
}

\begin{abstract}
Extra-adrenal paraganglioma of the retroperitoneum is a very rare neoplasm arising from cells of the primitive neural crest. We report a case of an 80 -years-old woman, who has been transferred to our neurology department with unsteady gait and ataxia, no abdominal pain, accidentally - as part of polyneuropathy screening - a retroperitoneal tumor situated at the aortic bifurcation was found in an ultrasound examination. At first our suggested diagnosis was a GIST, so we performed a CT guided core biopsy, the histology result was a paraganglioma, so we did a blood test, which showed elevated noradrenaline in plasma and normetanephrine in urinalysis. The patient showed no metastasis in PET CT Scan and was successfully treated by complete surgical removal of the tumor.
\end{abstract}

\section{Introduction}

Retroperitoneal neoplasms are rare tumors, easily misdiagnosed and present several therapeutic challenges because of their rarity and relatively late presentation. Paragangliomas are tumors that arise from extra-adrenal medullary neural crest derivatives. They are usually located in the head and neck but can be found in various body sites, including the chest cavity, abdomen, pelvis and bladder.

They can occur at any age, most commonly in young adults. The patient show a median age of $37-43$ years, the distribution between male and female patients is balanced $(1: 1)[1,2]$.

We report the case of an 80-years-old asymptomatic woman, with accidental diagnosis of a retroperitoneal paraganglioma.

\section{Case report}

We present a case of an 80-year old female patient, who was transferred to our neurology department with unsteady gait and ataxia. Patient's history was largely unremarkable, mild arterial hypertonus was well treated with an ACE-Inhibitor. There were no hypertensive crises in her anamnesis.

As part of a polyneuropathy screening an ultrasound examination of the abdomen and pelvis showed an unclear, heterogeneous lesion in the left abdominal cavity without organ reference.

Subsequent examination with CT showed an inhomogeneous, encapsulated, partially contrast enhancing tumor, with $8 \times 5 \mathrm{~cm}$ diameter. It was located retroperitoneal with close connection to the abdominal aorta and the inferior mesenteric artery. Especially in the arterial phase it showed a heterogeneous contrast-enhancement (Figure 1). Our suspected diagnosis at this time was a gastrointestinal stromal tumor (GIST). Also a PET-CT was performed. It showed a low FDGuptake with an SUV of 5,4. Furthermore, no metastatic lesions could be detected.

In consultation with colleagues from our neurology, surgery and internal medicine department, we decided to perform a CT-guided biopsy of the suspicious tumor (Figure 2).

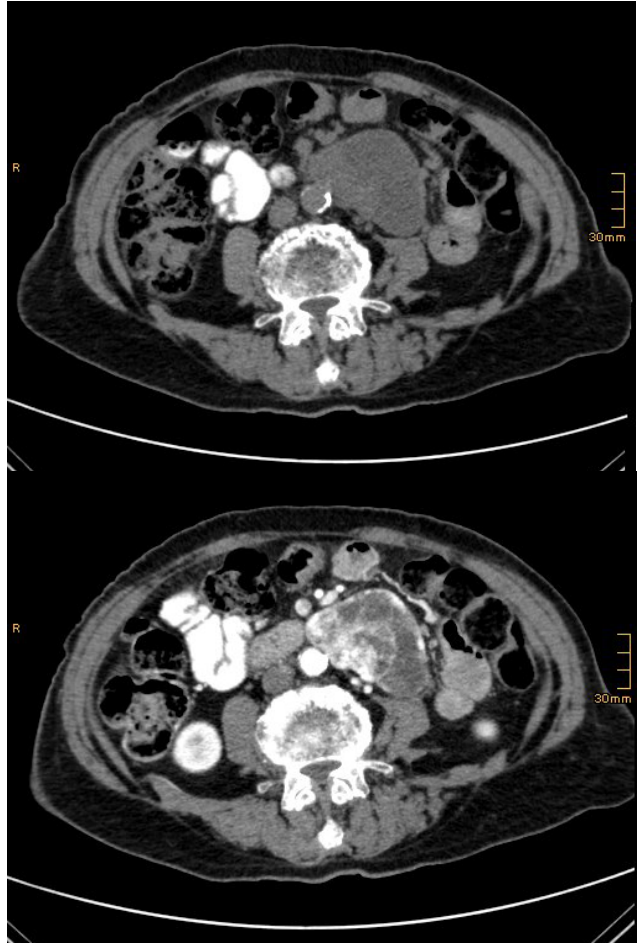

Figure 1. Computed tomography before (left) and after i.v. administration of contrast agent (right) shows an inhomogeneous, capsulated solid mass in the retroperitoneal cavity left to the abdominal aorta with strong arterial enhancement

${ }^{*}$ Correspondence to: Michael Hofko, Central Radiology Institute, Kepler University Hospital, Medical Faculty of the Johannes Kepler University, Linz, Austria, E-mail: micheal.hofko@kepleruniklinikum.at

Key words: paraganglioma, retroperitoneal, core biopsy, CT, PET-CT

Received: March 18, 2020; Accepted: April 15, 2020; Published: April 17, 2020 


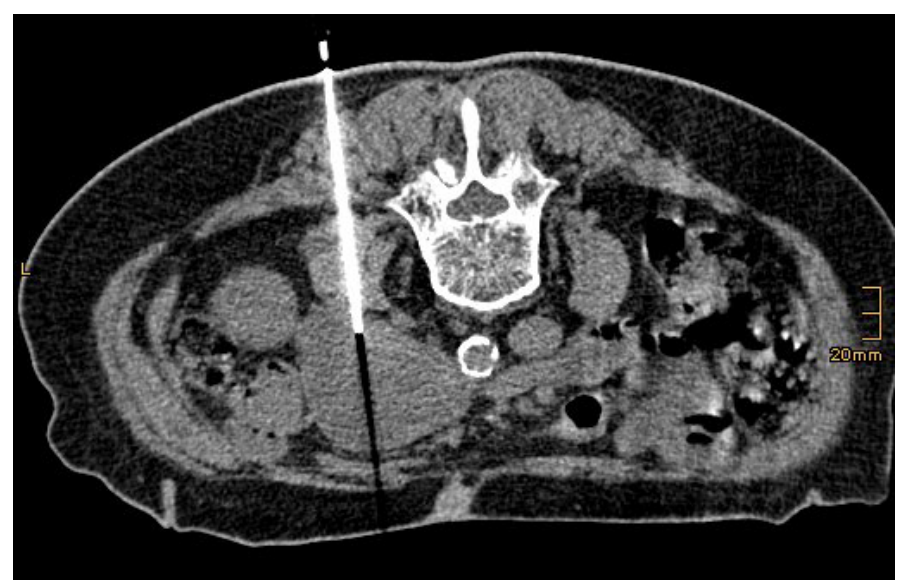

Figure 2. The picture shows the needle end position of CT-guided core biopsy in prone position

Histological workup resulted in the following: Blood coagulation and fibrous stroma with extensive portions of a small complexes and trabeculae in tumor tissue with relatively monomorphic cell elements with pale basophilic cytoplasm and roundish cell nuclei. In between partially fibrosed stroma. There were no high-grade pleomorphism of the tumor cells. Mitoses and necrosis are not visible. A strong antibody response against synaptophysin, chromogranin and (nuclear) with antibodies against GATA3 could be demonstrated. There was no reaction with antibodies against TTF1, Calretinin and DOG1, additional only a low proliferative activity (Ki67) in the area of 1-2\% of tumor cells. There was no evidence for a lymphoma or a GIST.

Because of the histologic result we decided to take a special blood analysis and urinalysis for catecholamines with the following results: $\mathrm{ml})$

Noradrenaline (blood plasma): 952,4 pg/ml (norm value: $<600$ pg/

Adrenaline (blood plasma): 93,2 pg/ml (norm value: $<100 \mathrm{pg} / \mathrm{ml}$ )

Metanephrine (urine): $153 \mu \mathrm{g} /$ day (norm value: $<350$ )

Normetanephrine (urine): $965 \mu \mathrm{g} /$ day (norm value: < 600)

Our final diagnosis was a retroperitoneal paraganglioma with no evidence of metastatic spread.

\section{Discussion}

Paragangliomas occur very rarely at any patients age, most commonly in young adults. The patients show a median age of $37-43$ years, the distribution between male and female patients is balanced $(1: 1)[1,2]$.

They are classified as a subgroup of tumors of the adrenal medulla und extra-adrenal paraganglia and were first described in 1953. The estimated annual incidence is 3 per million [3,4]. 2004 WHO classification of endocrine tumours defines phaeochromocytoma as a tumour arising from chromaffin cells in the adrenal medulla. Closely related tumours in extra-adrenal sympathetic and parasympathetic paraganglia are classified as extra-adrenal paraganglioma.[5]

Most common locations are the organ of the Zuckerkandl, the bladder wall, retroperitoneal, heart, mediastinum, carotid bodies and juxtaglomerular bodies [5]. The organ of Zuckerkandl is a chromaffin tissue derived from the neural crest, located at the aortic bifurcation or the origin of the inferior mesenteric artery [6]. About $10 \%$ of the retroperitoneal paraganglioma arise beside the adrenal gland [2].
Paragangliomas and pheochromocytomas can occur hereditary with a strong association to other genetic diseases like neurofibromatosis typ 1, multiple endocrine neoplasia (MEN $2 \mathrm{a} / 2 \mathrm{~b}$ ), von Hippel Lindau (vHL) and familial PGL syndrome [7].

In $50 \%$ the patients present with symptoms, such as palpitations, headache, sweating and hypertension caused by catecholamine secretion, the other half are nonfunctional paragangliomas. Retroperitoneal paragangliomas sometimes lead to nausea, vomiting, constipation and abdominal pain, sometimes the tumor causes ileus [8].

Often the diagnosis of non-functional paragangliomas is, because of absent clinical symptoms, delayed, so they pose a significant diagnostic challenge [2].

10 to $15 \%$ of paragangliomas can be malignant based on histologic analysis [9], but the only reliable criterion remains the presence of distant metastases. Metastasis of paraganglioma occur usually in the lungs, lymph nodes, liver, bones or the spleen. Most paragangliomas have an intact capsule with abundant blood vessels both on its surface and inside. Therefore, most paragangliomas can be removed without any complications [10].

A preoperative medication, e.g. Phenoxybenzamine - a very expensive option - is recommend in order to perform a safe resection. Alternative, a1-selective blockers (prazosin, doxazosin, terazosin), calcium channel blockers, angiotensin-converting enzyme (ACE) inhibitors and angiotensin receptor blockers, $\beta$-adrenergic blockers, and $\alpha 2$-agonists have been successfully used in preoperative management. Intraoperative vasodilators and esmolol are also recommended. [7].

Laparoscopic surgery is a safe curative method in non-metastatic situation, with a quick postoperative recovery and no negative influence on local recurrence. Another therapeutic option for malignant paragangliomas is radionuclide therapy with radioactive iodine coupled with MIBG [11] .

For patients with metastatic paraganglioma resection is not a curative therapeutic option, but patients may benefit from an operation because of decreasing levels of catecholamine surge. Furthermore, resection prevents abdominal and retroperitoneal complications of the tumor (i.e. acute abdomen by ruptured retroperitoneal paraganglioma [5]) and increases the efficacy of adjuvant systemic therapies. Other therapeutic options in cases of metastatic tumors are radiation therapy and ablative procedures [12].

\section{Conclusion}

Our case intends the need to consider paraganglioma of the retroperitoneum in the differential diagnosis of retroperitoneal tumours. Our first suspect was a GIST and so we carried out a CT guided core biopsy. Afterwards the blood and urine examination showed elevated noradrenaline and normetanephrine. PET CT scanning for metastasis was negative. Finally, our patient was successfully treated by complete surgical removal of the tumor.

Long-term follow-up is mandatory in those patients, because of the recurrence risk. Monitoring of hypertension and plasma or urine metanephrine levels are recommended (i.e. 10 days after operation and once a year for life). If the patient is asymptomatic, the screening can be based on a discussion with the patient and an informed decision could be made [3]. 


\section{Disclosure}

All co-authors do not report conflicts of interest.

\section{Grant/financial support}

No financial supports.

\section{References}

1. M. S. Belhamidi et al., "An unusual localization of retroperitoneal paraganglioma: A case report," Pan Afr. Med. J., 2015.

2. E. Gannan, P. Van Veenendaal, A. Scarlett, and M. Ng, "Retroperitoneal non-functioning paraganglioma: A difficult tumour to diagnose and treat," Int. J. Surg. Case Rep., vol. 17, pp. 133-135, Jan. 2015.

3. C. J. Neugarten, N. A. Sopko, D. Sundi, J.-J. Liu, and T. J. Bivalacqua, "Episodic Hypertension With an Adrenal Mass: A Red Herring q," 2014.

4. J. Turchini, V. K. Y. Cheung, A. S. Tischler, R. R. De Krijger, and A. J. Gill, "Pathology and genetics of phaeochromocytoma and paraganglioma," Histopathology, vol. 72, no. 1. Blackwell Publishing Ltd, pp. 97-105, 01-Jan-2018.
5. K. K. Yau, W. T. Siu, and M. K. W. L. Li, "Unusual cause of acute abdomen - Ruptured retroperitoneal paraganglioma," Asian J. Surg., 2008.

6. G. Klöppel, "Tumoren des Nebennierenmarks und der Paraganglien," Pathologe, vol. 24 , no. 4, pp. $280-286,2003$.

7. S. G. Tevosian and H. K. Ghayee, "Pheochromocytomas and Paragangliomas," Endocrinology and Metabolism Clinics of North America, vol. 48, no. 4. W.B. Saunders, pp. 727-750, 01-Dec-2019.

8. W. C. Lin, H. Y. Wang, C. W. Chang, J. L. Lin, and C. H. Tsai, "Retroperitoneal paraganglioma manifesting as paralytic ileus: A case report," J. Med. Case Rep., 2012.

9. S. Asa, S. Ezzat, and O. Mete, "The Diagnosis and Clinical Significance of Paragangliomas in Unusual Locations," J. Clin. Med., 2018.

10. J. He, X. Wang, W. Zheng, and Y. Zhao, "Retroperitoneal paraganglioma with metastasis to the abdominal vertebra: A case report," Diagn. Pathol., 2013.

11. P. Y. Cai, R. Golan, and B. Yanke, "Retroperitoneal Paraganglioma Involving the Renal Hilum: A Case Report and Literature Review," Urology, 2018.

12. S. Jasim and C. Jimenez, "Metastatic pheochromocytoma and paraganglioma: Management of endocrine manifestations, surgery and ablative procedures, and systemic therapies," Best Practice and Research: Clinical Endocrinology and Metabolism. 2019.

Copyright: (C2020 Hofko M. This is an open-access article distributed under the terms of the Creative Commons Attribution License, which permits unrestricted use, distribution, and reproduction in any medium, provided the original author and source are credited. 